\title{
XIII. An asymptotic formula for the hypergeometric function $\Delta_{4}(\mathrm{z})$
}

\section{Dorothy Wrinch M.Sc.}

To cite this article: Dorothy Wrinch M.Sc. (1921) XIII. An asymptotic formula for the hypergeometric function ${ }_{0} \Delta_{4}(\mathrm{z})$, Philosophical Magazine Series 6, 41:242, 161-173, DOI: 10.1080/14786442108636208

To link to this article: http://dx.doi.org/10.1080/14786442108636208

册 Published online: 08 Apr 2009.

Submit your article to this journal $\widetilde{3}$

Џ Article views: 2

Q

View related articles 주 
TH E

LONDON, KDINBURGH, AND DUBLIN

PHILOSOPHICAL MAGAZINE

\author{
AND \\ JOURNAL OF SCIENCE.
}

[8IXTH BERIE8.]

FE BRUARY 1921.

XIII. An Asymptotic Formula for the Hypergeometric Function ${ }_{0} \Delta_{4}(z)$. By Dorothy WRINCH, M.Sc., Fellow of Girton College, Cambridge, and Member of the Research Staff, University College, London *

THE present communication is concerned with the 1 asymptotic forms to which eertain generalized hypergeometric functions tend as their argument which may be real or complex is increased. The expansions obtained include as special cases most of the more familiar asymptotic expansions of the more special functions of hypergeometric type. The investigation originally arose in relation to certain functions which occux in physical problems, more especially those of elasticity, and the need for asymptotic values of these functions has been pointed out $\dagger$. The particular instance which gave rise to the investigation is concerried with the lateral vibrations of bars whose crosssection is a function of the distance from one end. The more important functions which thus arise can be included in a hypergeometric type analogous to the Bessel functions. We consider in this paper the general function

$$
\begin{aligned}
{ }_{0} \Delta_{4}[1+\alpha, 1+\beta, 1 & +\gamma, 1+\delta ; z] \\
& =1+\sum_{v=1}^{\infty} \frac{z_{\alpha \beta \gamma \delta}}{\Pi(1+\alpha)(2+\alpha) \cdots(v+\alpha)},
\end{aligned}
$$

where the parameters $\alpha, \beta, \gamma, \delta$ can take all values except

* Communicated by the Author.

$\dagger$ Vide e. g. Nicholson, Proc. Roy. Soc. 1917.

Phil. Mag. S. 6. Vol. 41. No. 242. Feb. 1921. 
negative integer values. The expansion of this function is developed for all values of $z$, real or complex, and the procedure is indicated by which we can determine the linear combination of solations of a characteristic differential equation of the fourth order which takes any of the individual forms

$$
\begin{aligned}
& e^{4 \dot{x}^{1 / 4}} x^{-\frac{1}{4}(\alpha+\beta+\gamma+\delta+3 / 2)}, \quad e^{-4 x^{1 / 4}} x^{-\frac{1}{4}(a+\beta+\gamma+\delta+3 / 2)}, \\
& x^{-\frac{1}{2}(\alpha+\beta+\gamma+\delta+3 / 2)} \cos \left[4 x^{1 / 4}-\frac{1}{4}(\alpha+\beta+\gamma+\delta+3 / 2) \pi / 2\right], \\
& x^{-\frac{1}{4}(a+\beta+\gamma+\delta+3 / 2)} \sin \left[4 x^{1 / 4}-\frac{1}{4}(\alpha+\beta+\gamma+\delta+3 / 2) \pi / 2\right], \\
& \text { near infinity. }
\end{aligned}
$$

Approximation to a Contour Integral.

Consider the integral

$$
\begin{aligned}
& \frac{1}{2 \pi i} \int\left(1+\frac{t}{1+\alpha}+\right.\left.\frac{t^{2}}{1+\alpha \cdot 2+\alpha} \cdots\right) \\
&\left(1+\frac{z / t}{1+\beta}+\frac{(z / t)^{2}}{1+\beta \cdot 2+\beta}+\ldots\right) \frac{d t}{t},
\end{aligned}
$$

$z$ being a complex number with principal argument $\phi$ and modulus $\rho$, round a circle enclosing the origin. Since both the series

$$
\begin{gathered}
{ }_{0} \Delta_{1}(t, 1+\alpha)=1+\frac{t}{1+\alpha}+\frac{t^{2}}{1+\alpha \cdot z+\alpha} \ldots, \\
{ }_{0} \Delta_{1}(z / t, 1+\beta)=1+\frac{z / t}{1+\beta}+\frac{z^{2} / t^{2}}{1+\beta \cdot 2+\beta} \cdots,
\end{gathered}
$$

are regular within the contour, the integral is equal to the residue of the integrand at the point $t=0$, viz. :

$$
\begin{aligned}
{ }_{0} \Delta_{2}(z ; 1+\alpha, 1+\beta) & =1+\frac{z}{1+\alpha \cdot 1+\beta} \\
& +\frac{z^{2}}{1+\alpha \cdot 2+\alpha \cdot 1+\beta \cdot 2+\beta}+\ldots
\end{aligned}
$$

Thus in order to approximate to ${ }_{0} \Delta_{2}(z ; 1+\alpha, 1+\beta)$ as $\rho \rightarrow \infty$, we may consider the asymptotic behaviour of the integral

$$
\mathrm{I}=\frac{1}{2 \pi i} \int_{c}{ }_{0} \Delta_{1}(t, 1+\alpha)_{0} \Delta_{1}(z / t, 1+\beta) \frac{d t}{t}
$$


Formula for the Hypergeometric Function ${ }_{0} \Delta_{4}(z) .163$ as $\rho \rightarrow \infty$ where $C$ is a circular contour round the point $t=0$. Let $\delta$ be the radius of tho circle $\mathrm{C}$, then

$$
\mathrm{I}=\int_{-\pi}^{\pi}{ }_{0} \Delta_{1}\left(\rho / \delta e^{i \phi-i \theta}, 1+\beta\right)_{0} \Delta_{1}\left(\delta e^{i \theta}, 1+\alpha\right) d \theta .
$$

Let $\chi$ stand for the argument of $z$, which is such that $-\pi<(\chi-\theta)<\pi$. We, can approximate to the value of $\mathrm{I}$, if $\delta$ is such that $\delta$ and $\rho^{\prime} \delta$ tend to infinity as $\rho \rightarrow \infty$, since

$$
{ }_{0} \Delta_{1}(z, 1+v)-\Gamma(1+v) e^{z_{i}}-v-\sum_{n=1}^{\infty} \frac{v \cdot v-1 \ldots v-n+1}{z^{n}}
$$$$
\text { If }-\pi<\chi<\pi \text { and arg } z^{-v}=-n \chi \text {, }
$$

where

$$
\mathrm{I} \sim \mathrm{I}_{1}-\mathrm{I}_{2}-\mathrm{I}_{3}+\mathrm{I}_{4},
$$

$$
\begin{aligned}
& \left.I_{1}=\Gamma(1+\alpha) \Gamma(1+\beta) \int_{-\pi}^{\pi} e^{\rho / \delta e^{i(x-\theta)}}\left[\rho / \delta e^{i(x-\theta}\right)\right]^{-\beta} \\
& \times e^{i e^{i \theta}}\left(\delta e^{i \theta}\right)^{-\alpha} d \theta \\
& \mathrm{I}_{2}=\int_{-\pi}^{\pi} e^{r / \delta e^{i(\gamma-\theta)}}\left(\rho / \delta e^{i(\chi-\theta)}\right)-\beta \sum_{n=1}^{\mathbb{N}} \frac{\alpha(\alpha-1) \ldots \alpha-n+1}{\left(\delta e^{i \theta}\right)^{n}} d \theta, \\
& \mathbf{I}_{3}=\int_{-\pi}^{\pi} e^{\delta e^{i \theta}}\left(\delta e^{i \theta}\right)-\alpha \sum_{n=1}^{N} \frac{\beta(\beta-1) \ldots(\beta-n+1)}{\left[\rho / \delta e^{i(\chi-\theta)}\right]^{n}} d \theta, \\
& I_{4}=\int_{-\pi}^{\pi} \sum_{n=1}^{N} \frac{\alpha(\alpha-1) \ldots(\alpha-n+1)}{\left(\delta e^{i \theta}\right)^{n}} \sum_{n=1}^{N} \frac{\beta(\beta-1) \ldots(\beta-n+1)}{\left[\rho / \delta e^{i(\chi-\theta)}\right]^{n}} .
\end{aligned}
$$

The first is an integral of an oscillatory exponential type, and we may approximate to its value by Kelvin's method*. "Critical points" of the integrand occur where the derivate of the exponent is zero, or

$$
\frac{l}{d \theta}\left(\frac{\rho}{\delta} e^{i(\chi-\theta)}+\delta e^{i \theta}\right)=0
$$

and therefore are easily seen to exist when and only when $\delta=\rho^{\frac{x}{2}}$. Taking this value for $\delta$, critical values of $\theta$ are given, $\mu$ being an integer, by

$$
\text { or } \quad \begin{aligned}
\chi-\theta & =\theta+2 \mu \pi \\
\theta & =\frac{\chi}{2}-\mu \pi .
\end{aligned}
$$

* A more rigorous investigation can be made by the "method of steepest descents." But this would be very long, and is not necessary for our purposes. 
Suppose $\chi_{r}$ is a critical point such that $-\pi<\chi_{r}<\pi$, then $\chi_{r}-\theta$ and $\theta$ both lie between $\pm \pi$, and since they differ by zero or a multiple of $2 \pi$ they must be equal.

Now as $|x| \rightarrow \infty$,

$$
\int_{0}^{x} e^{-z^{2}} d z \sim+\frac{1}{2} \sqrt{ } \pi \text { if }-\frac{\pi}{2}<\arg x<\frac{\pi}{2} .
$$

Hence as $|x| \rightarrow \infty$, if $a$ is positive and real,

Then

$$
\int_{0}^{a} e^{-x \epsilon^{2}} d \epsilon-\frac{1}{2} \sqrt{ } \pi, \text { if } \quad-\frac{\pi}{2}<\arg \sqrt{ } x<\frac{\pi}{2}
$$

$$
\begin{aligned}
& \rho^{-\frac{1}{2}(\alpha+\beta)} e^{-i \chi_{r}(\alpha+\beta)} \int_{-\pi-\chi_{r}}^{\pi-\chi_{r}} e^{\rho^{\frac{1}{2}} e^{i \chi_{r}\left(2-\epsilon^{2}\right)}} d \epsilon
\end{aligned}
$$

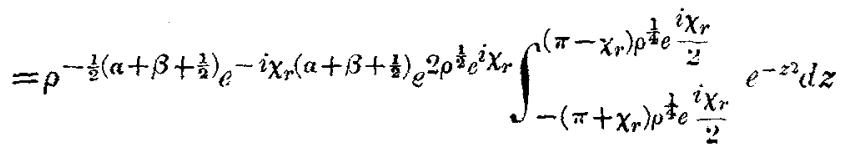

$$
\begin{aligned}
& \sim \sqrt{ } \pi \rho^{-\frac{1}{2}\left(a+\beta+\frac{1}{3}\right)} e^{-i \chi_{r}\left(\alpha+\beta+\frac{1}{3}\right)} e^{2 \rho^{\frac{1}{2}} e^{i} \chi_{r}}
\end{aligned}
$$

Next take the case when $\pi$ and therefore also $-\pi$ is a critical point; then the integrals

$$
\rho^{-\frac{1}{2}(\alpha+\beta)} \int_{-2 \pi}^{0} e-2 \rho^{\frac{1}{2}} \cos \epsilon d \epsilon,
$$

and

$$
\rho^{-\frac{1}{2}(\alpha+\beta)} \int_{0}^{2 \pi} e^{-2 \rho \frac{1}{2} \cos \varepsilon} d \epsilon,
$$

are negligible as $\rho \rightarrow \infty$, after the manner of the asymptotic theory of the function $I_{n}(x)$. Hence $\pm \pi$ as critical points contribute nothing to the asymptotic value of $\mathrm{I}_{1}$. Therefore

$$
\mathbf{I}_{1}-\Gamma(1+\alpha) \Gamma(1+\beta) \Sigma_{N} / \pi \rho^{-\frac{1}{2}\left(\alpha+\beta+\frac{1}{2}\right)} e^{-i X_{r}\left(\alpha+\beta+\frac{1}{2}\right)} e^{2 \rho^{\frac{1}{2}} e^{i} \chi_{r}},
$$

the summation being taken over all $\chi_{r}$ 's satisfying the conditions

$$
-\pi<\chi_{r}<\pi ; \quad-\chi_{r}=\frac{\chi}{2}-\mu \pi \text {. }
$$

Hence, as $\rho \rightarrow \infty$ if $-2 \pi<\phi<2 \pi$

$$
\begin{aligned}
& \mathrm{I}_{1} \sim \frac{\Gamma 1+\alpha \Gamma 1+\beta}{2 \sqrt{ } \pi} \rho^{-\frac{1}{2}\left(\alpha+\beta+\frac{1}{2}\right)}\left[e^{-i \frac{\phi}{2}\left(\alpha+\beta+\frac{1}{2}\right)}\right] \\
& \times\left[e^{2 p^{\frac{1}{2}} e^{\frac{i \phi}{2}}}+e^{-2 \rho^{\frac{2}{2} e} \frac{i \phi}{2}} e^{ \pm \pi i\left(\alpha+\beta+\frac{1}{2}\right)}\right],
\end{aligned}
$$


Formula for the Hyperyeometric Function ${ }_{0} \Delta_{4}(z) . \quad 165$ according as $\phi$ is positive or negative, the second term being evanescent when $\phi=0$.

Applying Kelvin's method to $I_{2}, I_{3}, I_{4}$, we see that no "critical points" exist. Hence the asymptotic equivalence stands in the form

$$
\begin{aligned}
1+\frac{z}{1+\alpha \cdot 1+\beta}+\cdot \frac{z^{2}}{1+\mu \cdot 2+\alpha \cdot 1+\beta \cdot 2+\beta}+\ldots \\
\quad \frac{\Gamma(1+\alpha) \Gamma(1+\beta)}{2 \sqrt{\pi}} \rho^{-\frac{1}{2}\left(\alpha+\beta+\frac{1}{2}\right)} e^{-\frac{i \phi}{2}\left(\alpha+\beta+\frac{1}{2}\right)} \\
\quad \times\left[\rho^{2 \rho^{\frac{1}{2}} e \frac{i \phi}{2}}+e^{-2 \rho^{\frac{1}{2}} e} e^{ \pm \pi i\left(\alpha+\beta+\frac{1}{2}\right)}\right]
\end{aligned}
$$

according as $\phi$ is positive or negative, the second term being evanescent when $\phi=0$.

In particular when $\rho$ is real

$$
\begin{array}{r}
1+\frac{\rho}{1+\alpha \cdot 1+\beta}+\ldots \sim \frac{\Gamma 1+\alpha \Gamma 1+\beta}{2 \sqrt{ } \pi} \rho^{-\frac{i}{2}\left(\alpha+\beta+\frac{1}{2}\right)} e^{2 \rho \frac{1}{2}}, \\
1-\frac{\rho}{1+\alpha \cdot 1+\beta} \cdots \sim \frac{\Gamma 1+\alpha \Gamma 1+\beta}{\sqrt{ } \pi} \rho^{-\frac{1}{2}\left(\alpha+\beta+\frac{1}{2}\right)} \\
\cos \left[2 \rho^{\frac{1}{2}}-\frac{\pi}{2}\left(\alpha+\beta+\frac{1}{2}\right)\right], \\
1+\frac{i \rho}{1+\alpha \cdot 1+\beta} \cdots \sim \frac{\Gamma 1+\alpha \Gamma 1+\beta}{\sqrt{ } \pi} \rho^{-\frac{1}{2}\left(\alpha+\beta+\frac{1}{2}\right)} e^{\frac{3}{8} \pi i\left(\alpha+\beta+\frac{1}{2}\right)} \\
\cos \left[2 \rho^{\frac{1}{3}}-\frac{5}{8} \pi\left(\alpha+\beta+\frac{1}{2}\right)\right], \\
1-\frac{i \rho}{1+\alpha \cdot 1+\beta} \cdots \sim \frac{\Gamma 1+\alpha \Gamma 1+\beta}{\sqrt{ } \pi} \rho^{-\frac{1}{2}\left(\alpha+\beta+\frac{1}{2}\right)} e^{-\frac{3}{8} \pi i\left(\alpha+\beta+\frac{1}{2}\right)} \\
\cos \left[2 \rho^{\frac{1}{2}}+\frac{5}{8} \pi\left(\alpha+\beta+\frac{1}{2}\right)\right]
\end{array}
$$

while, in general, if $\phi$ and $\rho$ are the principal argument and modulus of $z$ respectively:

$$
\begin{aligned}
& 1+\frac{z}{1+\alpha \cdot 1+\beta} \cdots-\frac{\Gamma 1+\alpha \Gamma 1+\beta}{\sqrt{ } \pi} e^{2 \rho^{\frac{1}{2}} \cos \frac{\phi}{2}} \\
& \times e^{ \pm \frac{\pi i}{2}(\alpha+\beta+1)-\frac{i \phi}{2}(\alpha+\beta+1)} \cos \left[2 \rho^{\frac{2}{2}} \sin \frac{\phi}{2} \mp \frac{\pi}{2}\left(\alpha+\beta+\frac{1}{2}\right)\right],
\end{aligned}
$$

the upper or lower signs being adopted according as $\phi$ is 
positive or negative, and

$$
1+\frac{z}{1+\alpha \cdot 1+\beta} \cdots \sim \frac{\Gamma 1+\alpha \Gamma 1+\beta}{2 \sqrt{ } \pi} \rho^{-\frac{1}{2}\left(\alpha+\beta+\frac{1}{2}\right)} e^{2 \rho^{\frac{1}{2}}},
$$

when $\phi$ is zero.

[It may be remarked that the form of this result contains an apparent ambiguity: for there are two values of $\phi$ such that

and

$$
-2 \pi<\phi<2 \pi
$$

$$
\arg (z)=\phi \text {. }
$$

Suppose $\phi_{1}$ and $\psi_{2}$ are values of $\phi$, satisfying these two conditions.

Then $\phi_{1} \sim \phi_{2}=2 \pi$, and

$$
\begin{aligned}
& e^{-i \phi_{1} / 2\left(\alpha+\beta+\frac{1}{2}\right)}\left[e^{2 \rho^{\frac{1}{2} e} e^{i \phi_{1} / 2}}+e^{-2 \rho^{\frac{1}{2}} e^{i \phi \frac{1}{2}}} e^{ \pm \pi i\left(\alpha+\beta+\frac{1}{2}\right)}\right] \\
& =e^{-\frac{i \phi_{2}}{2}\left(\alpha+\beta+\frac{1}{2}\right)}\left[e^{2 \rho^{\frac{1}{2}} \frac{i \phi}{2}}+e^{-2 \rho^{\frac{1}{2}} e^{i \phi_{2} / 2}} e^{\mp \pi i\left(\alpha+\beta+\frac{1}{2}\right)}\right],
\end{aligned}
$$

the upper: signs or lower signs being taken according as $\phi_{1}$ is positive and $\phi_{2}$ negative (when $\phi_{1}-\phi_{2}=2 \pi$ ), or $\phi_{1}$ is negative and $\phi_{2}$ positive (when $\phi_{2}-\phi_{1}=2 \pi$ ). The same result is then obtained whichever is taken of the two values of $\phi$, and the ambiguity is only apparent.]

\section{The more general series.}

We may adopt the same method in order to obtain the asymptotic equivalent of the convergent series

$$
\begin{aligned}
{ }_{0} \Delta_{4}[z ; & 1+\alpha, 1+\beta, 1+\gamma, 1+\delta] \\
& =1+\frac{z}{1+\alpha \cdot 1+\beta .1+\gamma \cdot 1+\delta}+\ldots,
\end{aligned}
$$

where the principal argument of $z$ is $\phi$ and its modulus is $\rho$.

For

$$
\begin{aligned}
{ }_{0} \Delta_{4}[z ; & 1+\alpha, 1+\beta, 1+\gamma, 1+\delta] \\
= & \frac{1}{2 \pi i} \int_{c}{ }_{0} \Delta_{2}[* / t ; 1+\alpha, 1+\beta]_{0} \Delta_{2}[t ; 1+\gamma, 1+\delta] \frac{d t}{t},
\end{aligned}
$$

$\mathrm{C}$ being a circle with its centre at the origin. We can again approximate to this integral as $|z| \rightarrow \infty$ by Kelvin's method. Let $\delta$ be the radius of the circle, then

$$
\begin{aligned}
& { }_{0} \Delta_{4}(z ; 1+\alpha, 1+\beta, 1+\gamma, 1+\delta) \\
& \quad=\frac{1}{2 \pi} \int_{-\pi}^{\pi}{ }_{0} \Delta_{2}\left[\rho / \delta e^{i(\phi-\theta)} ; 1+\alpha, 1+\beta\right]_{0} \Delta_{2}\left[\delta e^{i \theta} ; 1+\gamma, 1+\delta\right] d \theta .
\end{aligned}
$$


Formula for the Hypergeometric Function ${ }_{0} \Delta_{4}(z) . \quad 167$

If $\delta$ is so chosen that $\rho / \delta$ and $\delta \rightarrow \infty$ with $\rho$, and $\chi$ stands for an argument of $z$ which makes $-2 \pi<\chi-\theta<2 \pi$, then

$$
\begin{aligned}
& { }_{0} \Delta_{4}(z ; 1+\alpha, 1+\beta, 1+\gamma, 1+\delta) \sim \frac{\Gamma 1+\alpha \Gamma 1+\beta \Gamma 1+\gamma \Gamma 1+\delta}{8 \pi^{2}} \\
& \int_{-\pi}^{\pi}\left[\rho / \delta-^{-\frac{\left(\alpha+\beta+\frac{1}{2}\right)}{2}} \delta^{-\frac{\gamma+\delta+\frac{1}{2}}{2}}\left(e^{i \theta}\right)-\frac{\gamma+\delta+\frac{1}{2}}{2}\left(e^{i(\chi-\theta)}\right)-\frac{a+\beta+\frac{1}{2}}{2}\right. \\
& \times\left(e^{2 \delta^{\frac{1}{2}} e^{i \theta / 2}}+e^{-2 \delta^{\frac{1}{2}} e^{i \theta / 2} e^{ \pm i \pi}\left(\gamma+\delta+\frac{1}{2}\right)}\right) \\
& \times\left(e^{2(\rho / \delta)^{\frac{1}{2}} e^{i(\chi-\theta) / 3}}+e^{-2(\rho / \delta)^{\frac{2}{2}} e^{i(\chi-\theta) / 2}} e \pm i \pi\left(\alpha+\beta+\frac{1}{\sigma}\right) .\right.
\end{aligned}
$$

Either value of $\chi$ will give the same result, the order of the terms being interchanged. Let us take the smaller in absolute value, viz. $\phi$ itself.

In this expression the lower or upper sign in $e^{ \pm\left(\gamma+\delta+\frac{1}{2}\right) i \pi}$ is to be taken in the range in which $\theta$ is negative and that in which $\theta$ is positive respectively, and the upper or lower sign of $e^{ \pm \pi i\left(\alpha+\beta+\frac{1}{2}\right)}$ is to be taken in the range in which $\chi-\theta$ is positive and that in which $\chi-\theta$ is negative respectively. When $\theta=0$ the term $e^{-2 \delta^{\frac{1}{2}} e^{i \theta / 2}} e^{ \pm \pi i\left(\gamma+\delta+\frac{1}{2}\right)}$ is to be omitted. Similarly, when $\chi-\theta=0$, the term $e^{-2 \delta^{\frac{3}{3} e^{i \theta / 2}}} e^{ \pm i \pi\left(\alpha+\beta+\frac{1}{2}\right)}$ is to be omitted.

Hence

$$
\begin{aligned}
{ }_{0} \Delta_{4}(z ; 1+\alpha, 1+\beta, 1+\gamma, 1+\delta) \\
\\
\sim \frac{\Gamma 1+\alpha \Gamma 1+\beta \Gamma 1+\gamma \Gamma 1+\delta}{8 \pi^{2}}\left[{ }_{1} \mathrm{~N}_{1}+{ }_{1} \mathrm{~N}_{2}+{ }_{2} \mathrm{~N}_{1}+{ }_{2} \mathrm{~N}_{2}\right]
\end{aligned}
$$

where

$$
\begin{aligned}
& { }_{1} \mathrm{~N}_{1}=\int_{-\pi}^{\pi}\left(\delta e^{i \theta}\right)^{-\frac{\gamma+\delta+\frac{\gamma}{2}}{2}}\left(\rho / \delta e^{i \overline{\chi-\theta}}\right)-\frac{\alpha+\beta+\frac{1}{2}}{2} \\
& \times e^{2\left[i^{\frac{1}{2}} e^{i \theta / 2}+(\rho / \delta)^{\frac{\pi}{2}} e^{\frac{i \bar{\chi}-\bar{\theta}}{2}}\right]} d \theta, \\
& { }_{2} \mathrm{~N}_{1}=\int_{-\pi}^{\pi} e^{ \pm i \pi\left(\gamma+\delta+\frac{1}{2}\right)}\left(\delta e^{i \theta}\right)^{-\frac{\gamma+\delta+\frac{1}{2}}{2}} \\
& \times\left(\rho / \delta e^{i(\chi-\theta)}\right)^{-\frac{\alpha+\beta+\frac{1}{2}}{2}} e^{-2 \delta^{\frac{1}{2}} e^{i \theta / 2}+2(\rho / \delta)^{\frac{1}{2}} e^{i(\chi-\theta) / 2}} d \theta,
\end{aligned}
$$




$$
\begin{aligned}
& { }_{1} \mathrm{~N}_{2}=\int_{-\pi}^{\pi} e^{ \pm i \pi\left(\alpha+\beta+\frac{1}{2}\right)}\left(\delta e^{i \theta}\right)-\frac{\gamma+\delta+\frac{1}{2}}{2} \\
& \times\left(\rho / \delta e^{i(\chi-\theta)}\right){ }^{-\frac{a+\beta+\frac{\lambda}{2}}{2}} e^{2 \delta^{\frac{2}{2}} e^{i \theta / 2}-2(\rho / r)^{\frac{1}{2}} e^{i \bar{i}-\theta / 2}} d \theta, \\
& { }_{2} \mathrm{~N}_{2}=\int_{-\pi}^{\pi} e^{ \pm \pi\left(\gamma+\delta+\frac{1}{2}\right)} e^{ \pm \pi i\left(\alpha+\beta+\frac{1}{2}\right)}\left(\delta e^{i \theta}\right)^{-\frac{\gamma+\delta+\frac{1}{2}}{2}} \\
& \times\left(\rho / \delta e^{i(x-\theta)}\right)^{-\frac{\alpha+\beta+\frac{1}{2}}{2}} e^{-2 \delta^{\frac{1}{2}} e^{i \theta / 2}-2(\rho / \delta)^{\frac{1}{2}} e^{i(x-\theta) / 2}} d \theta .
\end{aligned}
$$

$W_{\theta}$ are still at liberty to choose $\delta$ as we please, provided only that $\rho / \delta$ and $\delta$ tend to infinity with $\rho$, the factors $e^{ \pm \pi i\left(\gamma+\delta+\frac{2}{2}\right)}$ being adopted according as $\theta$ is positive or negative, and the factors $e^{ \pm \pi i(a+\beta+1)}$ being adopted according as $\phi-\theta$ is positive or negative.

We shall therefore, if possible, choose $\delta$ with a view to ensuring the existence of critical points for the integrals ${ }_{1} \mathrm{~N}_{1},{ }_{1} \mathrm{~N}_{2},{ }_{2} \mathrm{~N}_{1},{ }_{2} \mathrm{~N}_{2}$.

Critical values of $\theta$ will exist if there exist roots of the two equations

$$
\frac{d}{d \theta}\left[\delta^{\frac{1}{2}} e^{\frac{i \theta}{2}} \pm\left(\frac{\rho}{\delta}\right)^{\frac{\pi}{2}} e^{i \frac{\theta-\theta}{2}}\right]
$$

We therefore choose $\delta=p^{\frac{2}{2}}$, which gives the only possible case.

Consider first the integrals ${ }_{1} \mathrm{~N}_{1}$ and ${ }_{2} \mathrm{~N}_{2}$. Critical values are given by the equation

$$
\theta=\frac{\phi}{2}+2 \mu \pi \text {. }
$$

There will be one, and only one, for each integral, and it will be

$$
\theta=\frac{\phi}{2} \text {. }
$$

Hence, using Stokes's result as before,

$$
\begin{aligned}
& { }_{1} \mathrm{~N}_{1} \sim \beta^{-i(\alpha+\beta+\gamma+\delta+1)} e^{-i \frac{\phi}{4}(\alpha+\beta+\gamma+\delta+1)} \\
& \times \int_{-\pi-\frac{\phi}{2}}^{\pi-\frac{\phi}{2}} e^{2 \rho^{t} e^{\frac{i \phi}{4}}}\left(2-\frac{\varepsilon^{2}}{4}\right) d \varepsilon \\
& =\sqrt{2 \pi} \cdot \rho^{-\frac{1}{4}(\alpha+\beta+\gamma+\delta+3 / 2)} e^{-i \frac{\phi}{4}(\alpha+\beta+\gamma+\delta+3 / 2)} e^{4 \rho^{\frac{1}{2}} e^{i \phi / 4}}
\end{aligned}
$$


Formula for the Hypergeometric Function ${ }_{0} \Delta_{4}(z) . \quad 169$

$$
\begin{aligned}
& { }_{2} \mathrm{~N}_{2} \sim \rho^{-\frac{1}{(2+\beta+\beta+\gamma+\delta+1)}\left(e^{i \frac{\phi}{2}}\right)^{-\frac{a+\beta+\gamma+\hat{j}+1}{2}} \pm \pi i(\gamma+\delta+a+\beta+1)} \\
& \times \int_{-\pi-\frac{\phi}{2}}^{\pi-\frac{\phi}{2}} e^{-2 \rho^{\frac{1}{4}} e^{i \frac{\phi}{4}}\left(2-\frac{\epsilon^{2}}{4}\right)} d \epsilon
\end{aligned}
$$

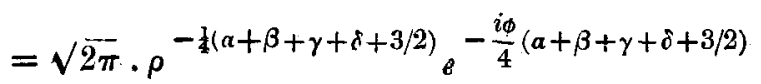

$$
\begin{aligned}
& \times e^{-4 \rho^{\frac{1}{2}} e^{\frac{i \phi}{4}}} e^{ \pm \pi i(\alpha+\beta+\gamma+\delta+3 / 2)},
\end{aligned}
$$

according as $\phi$ is positive or negative.

If $\phi=0$ the integral does not occur.

Consider next the integrals ${ }_{2} \mathrm{~N}_{1}$ and ${ }_{1} \mathrm{~N}_{2}$. Critical values are given by the equation

$$
\theta=\frac{\phi}{2}+(2 \mu+1) \pi ; \quad \phi-\theta=\frac{\phi}{2}-(2 \mu+1) \pi .
$$

There will be one, and one only, for each integral, viz.,

$$
\theta=\frac{\phi}{2} \pm \pi \text {, }
$$

according as $\phi$ is positive or negative, except in the case $\phi=0$, when there will be critical values $\pi$ and $-\pi$ if

$$
\theta=\frac{\phi}{2} \mp \pi, \quad \chi-\theta=\frac{\phi}{2} \pm \pi \text {. }
$$

Hence, according as $\phi$ is positive or negative,

$$
\begin{aligned}
& { }_{2} \mathrm{~N}_{1} \sim \rho^{-\frac{1}{4}(\alpha+\beta+\gamma+\delta+1)} \int_{-\pi-\left[\frac{\phi}{2} \mp \pi\right]}^{\pi-\left(\frac{\phi}{2} \mp \pi\right)} e^{\mp\left(\gamma+\delta+\frac{1}{2}\right) \iota \pi} \\
& \times e^{-i\left(\frac{\phi}{2} \mp \pi\right) \frac{\gamma+\delta+\frac{1}{2}}{2}} e^{-i\left(\frac{\phi}{2} \pm \pi\right) \frac{a+\beta+\frac{1}{2}}{2}}
\end{aligned}
$$

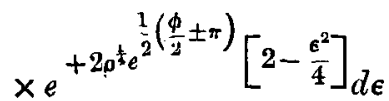

$$
\begin{aligned}
& =e^{4 \rho^{\frac{1}{2}} e^{\frac{1}{2}\left(\frac{\phi}{2} \pm \pi\right)}} \rho^{-\frac{\delta}{4}(\alpha+\beta+\gamma+\delta+1)} e^{-i\left(\frac{\phi}{2} \pm \pi\right) \frac{(\alpha+\beta+\gamma+\delta+1)}{2}} \\
& \int_{-\pi-\left(\frac{\phi}{2} \mp \pi\right)}^{\pi-\left(\frac{\phi}{2} \mp \pi\right)} e^{\left.-\frac{1}{2} \epsilon^{2} \rho^{\frac{1}{t} e^{\frac{1}{2}}\left(\frac{\phi}{2} \pm \pi\right.}\right)} d \epsilon, \\
& \sim \sqrt{ } 2 \pi\left[\rho e^{i(\phi / 2 \pm \pi)}\right]^{-\frac{1}{4}(\alpha+\beta+\gamma+\delta+3 / 2)} e^{\left.4 \rho \frac{1}{2} e^{i(\phi / 4 \pm \pi / 2}\right)}, \\
& \text { if } \phi \neq 0 \text {. }
\end{aligned}
$$


The values of $\phi$ lie between $-\pi$ and $+\pi(+\pi$ not excluded), and the result therefore holds in all cases under consideration, for Stokes's result holds for all integrals of the form $\int_{0}^{b} e^{-a z^{2}} d z$, when $-\pi<\arg a<\pi$.

Similarly,

$$
\begin{aligned}
& { }_{1} \mathrm{~N}_{2} \sim \sqrt{2 \pi} \rho-\frac{1}{4}(\alpha+\beta+\gamma+\delta+3 / 2) \\
& \times e^{-i\left(\frac{\phi}{2} \mp \pi\right) \frac{\alpha+\beta+\gamma+\delta+3 / 2}{2}} e^{\left.4 \rho^{\frac{1}{\beta}} e^{i\left(\frac{\phi}{4} \mp\right.} \frac{\pi}{2}\right)}
\end{aligned}
$$

If $\phi=0$,

$$
\begin{aligned}
& { }_{1} \mathrm{~N}_{2}={ }_{2} \mathrm{~N}_{1} \sim \frac{1}{2} \sqrt{2 \pi \rho}-\frac{3}{4}(\alpha+\beta+\gamma+\delta+3 / 2) \\
& \times e^{-\frac{i \pi}{2}(\alpha+\beta+\gamma+\delta+3 / 2)} e^{4 \rho^{\frac{1}{3} e}+\frac{\pi i}{2}} \\
& +\frac{1}{2} \sqrt{2 \pi} \rho^{-\frac{1}{2}(\alpha+\beta+\gamma+\delta+3 / 2)} \\
& \times e^{+\frac{i \pi}{2}(\alpha+\beta+\gamma+\delta+3 / 2)} e^{4 \rho^{\frac{2}{2} e} e^{-\frac{\pi i}{2}}}
\end{aligned}
$$

Hence

$$
\begin{aligned}
{ }_{1} \mathrm{~N}_{2}+{ }_{2} \mathrm{~N}_{1} \sim & \sqrt{2 \pi} \rho^{-\frac{1}{4}(\alpha+\beta+\gamma+\delta+3 / 2)} \\
& \times \cos \left[4 \rho^{\frac{1}{2}}-\frac{\pi}{2}(\alpha+\beta+\gamma+\delta+3 / 2] .\right.
\end{aligned}
$$

Thus if $-\pi<\phi \leq \pi$,

$$
\begin{gathered}
{ }_{1} \mathrm{~N}_{1}+{ }_{1} \mathrm{~N}_{2}+{ }_{2} \mathrm{~N}_{1}+{ }_{2} \mathrm{~N}_{2} \sim \sqrt{2 \pi} \rho-1(\alpha+\beta+\gamma+\delta+3 / 2) \\
{\left[e^{-\frac{i \phi}{4}(a+\beta+\gamma+\delta+3 / 2)} e^{4 \rho^{\frac{3}{3}} e^{\frac{i \phi}{4}}}\right.} \\
+e^{ \pm \frac{i \pi}{2}(\alpha+\beta+\gamma+\delta+3 / 2)} e^{4 \rho^{\frac{1}{4} e}-\frac{i \pi}{2}+\frac{i \phi}{4}} \\
+e^{-\frac{i \pi}{2}(\alpha+\beta+\gamma+\delta+3 / 2)} e^{4 \rho^{\frac{1}{4} e} \frac{\pi}{2}+\frac{\omega \phi}{4}} \\
\left.+e^{ \pm \pi i(\alpha+\beta+\gamma+\delta+3 / 2)} e^{-4 \rho^{\frac{1}{4}} e^{\frac{\delta \phi}{4}}}\right],
\end{gathered}
$$

according as $\phi$ is positive or negative, the last term being ouitted if $\phi$ is zero. 
Formula for the Hypergeometric Function ${ }_{0} \Delta_{4}(z) . \quad 171$

Hence $\quad{ }_{0} \triangle_{4}(\approx ; 1+\alpha, 1+\beta, 1+\gamma, 1+\delta)$

$$
\frac{\sim \frac{\Gamma 1+\alpha \Gamma 1+\beta \Gamma 1+\gamma \Gamma 1+\delta}{2^{5 / 2} \pi^{3 / 2}}}{\rho^{-\frac{1}{4}(\alpha+\beta+\gamma+\delta+3 / 2)}[\mathrm{B}]}
$$

where $\mathbf{B}$ is the preceding large bracket, according as $\phi$ is positive or negative, the last ter'm being omitted if $\phi$ is zero.

Hence in particular, $\rho$ being real,

$$
\begin{gathered}
{ }_{0} \Delta_{4}(\rho ; 1+\alpha, 1+\beta, 1+\gamma, 1+\delta) \\
=-1+\frac{\rho}{1+\alpha 1+\beta \cdot 1+\gamma \cdot 1+\delta} \cdots \\
\sim \frac{\Gamma 1+\alpha \Gamma 1+\beta \Gamma 1+\gamma \Gamma 1+\delta}{2^{5 / 2} \pi^{3 / 2}} \rho^{-\frac{1}{4}(\alpha+\beta+\gamma+\delta+3 / 2)} \\
{\left[e^{4 \rho^{\frac{1}{4}}}+2 \cos \left\{4 \rho^{\frac{1}{2}}-\frac{\pi}{2}(\alpha+\beta+\gamma+\delta+3 / 2)\right\}\right]}
\end{gathered}
$$

and

$$
\begin{aligned}
& 1-\frac{\rho}{1+\alpha \cdot 1+\beta \cdot 1+\gamma \cdot 1+\delta}+\ldots \\
& \sim \frac{\Gamma 1+\alpha \Gamma 1+\beta \Gamma 1+\gamma \Gamma 1+\delta}{2^{5 / 2} \pi^{3 / 2}}\left(e^{4 \rho^{\frac{1}{4}} \cos \frac{\pi}{4}}\right. \\
& \left.\quad \times \cos \left[4 \rho^{\frac{1}{4}} \sin \frac{\pi}{4}-\frac{\pi}{4}(\alpha+\beta+\gamma+\delta+3 / 2)\right]\right) .
\end{aligned}
$$

Special forms near infinity.

Series of the type ${ }_{0} \Delta_{4}$ occur as solutions of equations of the type

$$
\vartheta(\vartheta+a)(\vartheta+b)(\vartheta+c) \omega=z \omega,
$$

which are of frequent occurrence in problems of lateral vibrations of bars whose section is not uniform.

If we put

the equation becomes

$$
\omega=z^{-v} y \text {, }
$$

$$
(\vartheta-v)(\vartheta+a-v)(\vartheta+b-v)(\vartheta+c-v) y=z y .
$$

The general solution of the original equation-if we formally exclude the cases when logarithmic solutions are involved- 
is therefore, $\mathrm{P}, \mathrm{Q}, \mathrm{R}, \mathrm{S}$ being constants,

$$
\begin{aligned}
\mathrm{P}_{0} \Delta_{4}(1+ & a, 1+b, 1+c, 1 ; z) \\
& +z^{-a} Q_{0} \Delta_{4}(-a, 1+b-a, 1+c-a, 1 ; z) \\
& +z^{-b} \mathrm{R}_{0} \Delta_{4}(-b, 1+c-b, 1+a-b, 1 ; z) \\
& +z^{-c} \mathrm{~S}_{0} \Delta_{4}(-c, 1+a-c, 1+b-c, 1 ; z) .
\end{aligned}
$$

Let $y_{1} y_{2} y_{3} y_{4}$ represent these four solutions, which together form the general solution. Then the results obtained with respect to the asymptotic behaviour of

give

$$
{ }_{0} \Delta_{4}(1+\alpha, 1+\beta, 1+\gamma, 1+\delta ; z)
$$

$$
\begin{array}{r}
y_{1} \sim \frac{\Gamma 1+a \Gamma 1+b \Gamma 1+c}{2^{5 / 2} \pi^{3 / 2}} z^{-\frac{a+b+c+3 / 2}{4}}\left[e^{4 z^{\frac{1}{4}}}+e^{4 i z^{\frac{1}{4}}} e^{-\frac{\pi i}{2}(a+b+c+3 / 2)}\right. \\
\left.+e^{-4 i z^{\frac{1}{4}}} e^{+\frac{\pi i}{2}(a+b+c+3 / 2)}+e^{-4 z^{\frac{1}{4}}} e^{\mp \pi i(a+b+c+3 / 2)}\right],
\end{array}
$$$$
y_{2} \sim \frac{\Gamma 1-a \Gamma 1+b-a \Gamma 4 c-a}{2^{3 / 2} \pi^{3 / 2}} z^{-\frac{a+b+c+3 / 2}{4}}\left[e^{4 z^{\frac{1}{4}}}+e^{4 i z^{\frac{1}{4}}} e^{-\frac{i \pi}{2}(-3 a+b+c+3 / 2)}\right.
$$

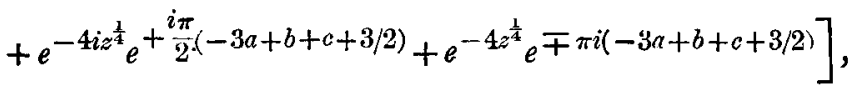

$$
\begin{gathered}
y_{3} \sim \frac{\Gamma 1-b \Gamma 1+c-b \Gamma 1+a-b}{2^{5 / 2} \pi^{3 / 2}} z^{-\frac{a+b+c+3 / 2}{4}}\left[e^{4 z^{\frac{1}{4}}}+e^{4 i z^{\frac{1}{4}}} e^{-\frac{i \pi}{2}(c+3 / 2+a-3 b)}\right. \\
+\ldots], \\
y_{4} \sim \frac{\Gamma 1-a \Gamma 1+a-c \Gamma 1+b-c}{2^{8 / 2} \pi^{3 / 2}} z^{-\frac{a+b+c+3 / 2}{4}}[\ldots] .
\end{gathered}
$$

Linear combinations of $y_{1} y_{2} y_{3} y_{4}$ therefore exist which behave asymptotically near infinity like

$$
\begin{aligned}
& z^{-\frac{(a+b+c+3 / 2)}{4} e^{z^{\frac{1}{4}}},} \quad z^{-\frac{a+b+c+3 / 2}{4}} e^{-4 z^{\frac{1}{4}}}, \\
& z^{-\frac{a+b+c+3 / 2}{4} \cos 4 z^{\frac{1}{4}}, \text { or } \quad z^{-\frac{a+b+c+3 / 2}{4}} \sin 4 z^{\frac{1}{4}} .}
\end{aligned}
$$

Let us consider the linear combination which is asymptotically equivalent to

$$
z^{-\frac{a+b+c+3 / 2}{4}} e^{-4 z^{\frac{1}{4}}}
$$


Formula for the Hypergeometric Function ${ }_{0} \Delta_{4}(z)$.

Writing

$$
\begin{aligned}
& y_{1}^{\prime}=\frac{2^{5 / 2} \pi^{3 / 2}}{\Gamma(1+a) \Gamma(1+b) \Gamma(1+c)} z^{\frac{a+b+c+3 / 2}{4}} y_{1}, \\
& y_{2}^{\prime}=\frac{2^{5 / 2} \pi^{3 / 2}}{\Gamma(1-a) \Gamma(1+b-a) \Gamma(1+c-a)} z^{\frac{a+b+c+3 / 2}{4}} y_{2}, \\
& y_{3}^{\prime}=\frac{2^{5 / 2} \pi^{3 / 2}}{\Gamma(1-b) \Gamma(1+c-b) \Gamma(1+a-b)} z^{\frac{a+b+c+3 / 2}{4} y_{3},} \\
& y_{4}^{\prime}=\frac{2^{5 / 2} \pi^{3 / 2}}{\Gamma(1-c) \Gamma(1+a-c) \Gamma(1+b-c)} z^{\frac{a+b+c+3 / 2}{4}} y_{4} ;
\end{aligned}
$$

it is clear that the linear combination

$$
\left|\begin{array}{cccc}
y_{1}^{\prime} & 1 & 1 & 0 \\
y_{2}^{\prime} & 1 & \cos \pi a & \sin \pi a \\
y_{3}^{\prime} & 1 & \cos \pi b & \sin \pi b \\
y_{4}^{\prime} & 1 & \cos \pi c & \sin \pi c
\end{array}\right|
$$

will behave asymptotically like $e^{-4 z^{\frac{1}{4}}}$. A simpler form of this expression is, on development,

$$
\begin{aligned}
& 4 y_{1}^{\prime} \sin (\pi b-c) \sin (\overline{\pi c-a}) \sin (\overline{\pi a-b}) \\
& -4 y_{2}{ }^{\prime} \sin \pi c \sin \pi b \sin \overline{\pi(b-c)} \\
& -4 y_{3}{ }^{\prime} \sin \pi a \sin \pi c \sin \pi(c-a) \\
& -4 y_{4}^{\prime} \sin \pi b \sin \pi a \sin \overline{\pi(a-b)} \\
& =-\frac{4}{\pi^{3}} \sin (\pi \overline{b-c}) \sin (\pi c-a) \sin \overline{\pi(a-b)} \sin \pi a \sin \pi b \\
& \sin \pi c\left[y_{1}{ }^{\prime} \Gamma(-a) \Gamma(-b) \Gamma(-c) \Gamma(1+a) \Gamma(1+b) \Gamma(1+c)\right. \\
& +y_{2} \Gamma(a) \Gamma(1-a) \Gamma(a-c) \Gamma(a-b) \Gamma(1+c-a) \Gamma(1+b-a) \\
& +y_{3}^{\prime} \Gamma(b) \Gamma(1-b) \Gamma(b-a) \Gamma(b-c) \Gamma(1-b+a) \Gamma(1-c+b) \\
& +y_{4}^{\prime} \Gamma(c) \Gamma(1-c) \Gamma(c-b) \Gamma^{\prime}(c-a) \Gamma(1-c+b) \Gamma(1-a+c) \text {. }
\end{aligned}
$$

Thus

$$
\begin{aligned}
& y_{1} \Gamma(-a) \Gamma(-b) \Gamma(-c)+y_{2} \Gamma(a) \Gamma(a-c) \Gamma(a-b) \\
& +y_{3} \Gamma(b) \Gamma(b-a) \Gamma(b-c)+y_{4} \Gamma(c) \Gamma(c-a) \Gamma(c-b) \\
& \quad \sim \lambda z^{-\frac{a+b+c+3 / 2}{4}} e^{-4 z^{\frac{1}{4}}},
\end{aligned}
$$

where $\lambda$ is a function only of $a, b$, and $c$.

In the same manner we can isolate the solutions which tend to the other individual asymptotic forms, and the value of $\lambda$ is obtained readily in each case. Such determinations are, however, apart from our main object, and this instance will suffice. 